\title{
Preface: Cosmic Chemical Evolution
}

Questions concerning the nature and origin of the chemical elements of which matter in the Universe is composed are as old as human inquiry. The history of the Universe itself is indeed written in the elemental and isotopic composition of its diverse components: galaxies, stars, interstellar gas in galaxies, the intergalactic medium, cosmic rays, and gas clouds at high redshifts. Since distinctive abundances and abundance patterns are characteristic of nucleosynthesis occurring in stars of different masses and corresponding lifetimes, interesting constraints on the star formation and nucleosynthesis histories may be contained in the abundance histories of both halo population stars and QSO absorption line systems. Using both distant quasars and most recently gamma-ray bursts, the star formation and abundance history of the high redshift Universe can also be probed. Significant progress has occurred in recent years as a consequence of a wealth of new information on cosmic phenomena - the spectroscopic and photometric properties of gas clouds and galaxies at high redshifts - pouring in from new ground and space based observatories, of improvements in our knowledge of the nuclear physics that dictates the character of explosive nucleosynthesis processes, and of rapidly expanding computational facilities which make possible detailed and more realistic modeling of stellar evolution and supernova nucleosynthesis.

The Universe emerged from an exciting first three minutes with a composition consisting of hydrogen $\left({ }^{1} \mathrm{H}\right)$, helium $\left({ }^{4} \mathrm{He}\right)$, deuterium $\left({ }^{2} \mathrm{D}\right),{ }^{3} \mathrm{He}$, and ${ }^{7} \mathrm{Li}$. Further synthesis of heavy elements then awaited the formation and evolution of the first stars, some $10^{8}$ to $10^{9}$ years later. The characteristics of the first stellar contributions to nucleosynthesis, whether associated with Population II or a Population III, reflect (as might be expected) the nucleosynthesis products of the evolution of massive stars $\left(\mathrm{M} \gtrsim 10 \mathrm{M}_{\odot}\right)$ of short lifetimes $(\tau \lesssim$ $10^{8}$ years $)$. Metal deficient stars $([\mathrm{Fe} / \mathrm{H}] \lesssim-3)$ in our own galaxy's halo show two significant variations with respect to solar abundances: the elements in the mass range from oxygen to calcium are overabundant - relative to ironpeak nuclei - by a factor $\approx 2-3$, and the abundance pattern in the heavy element regime $\mathrm{A} \gtrsim 60-70$ reflects the r-process abundance distribution that is characteristic of solar system matter, with no evidence for an s-process nucleosynthesis contribution. Both of these features are entirely consistent with nucleosynthesis expectations for massive stars and associated Type II supernovae. The introduction of both the s-process products of the evolution 
of low mass stars $\left(\mathrm{M} \approx 1-3 \mathrm{M}_{\odot}\right)$ and of iron nuclei from Type Ia supernovae occurs on a significantly longer timescale. In our Galaxy, evidence for low mass star input is evidenced at a metallicity $-2.5 \lesssim[\mathrm{Fe} / \mathrm{H}] \lesssim-2$, while the products of Type Ia supernovae seem first to have appeared at $[\mathrm{Fe} / \mathrm{H}] \sim-1$. Such trends are consistent as well with abundance determinations for both galactic globular clusters and nearby dwarf galaxies. They are also consistent with observations of the abundances and of the metallicity evolution (in the high redshift Universe) of damped Lyman- $\alpha$ systems.

The considerations noted above have provided the motivation and the setting for IAU Symposium 187 on "Cosmic Chemical Evolution." The current level of activity and excitement is reflected in and captured by the diverse papers that have been collected into this volume. Indeed, symposium papers address virtually all aspects of the problem of nucleosynthesis, galactic chemical evolution, and cosmic chemical evolution. Theoretical models of nucleosynthesis in the Big Bang, in the first stellar generations, and in stars and supernovae over the histories of our Galaxy and other galaxies provide a measure of element production over the lifetime of the Universe. Observational studies of abundances in stars, galaxies, gas concentrations in galaxies and clusters of galaxies, the intergalactic medium, and gas clouds at high redshift are reviewed. To the extent that heavy element nucleosynthesis is dominated by stellar and supernova contributions, this abundance data provides a quantitative measure of the level of star formation activity as a function of time (redshift) that seems quite consistent with the starlight history of the Universe and reionization. Finally, studies of the chemical evolution of galaxies and expectations for the chemical evolution of the Universe itself provide a firm basis for comparison of theory and observation. 